\title{
Is liver resection still required for patients who have predictive factors for complete pathologic necrosis after downstaging for locally advanced hepatocellular carcinoma?
}

\author{
Munseok $\mathrm{CHOl}^{1}$, Gi Hong $\mathrm{CHOI}^{* 2}$, Dai Hoon $\mathrm{HAN}^{2}$, Kyung Sik $\mathrm{KIM}^{2}$, Jin Sub $\mathrm{CHOl}^{2}$, \\ Beom Kyung $\mathrm{KIM}^{3}$, Seung Up KIM${ }^{3}$, Do Young $\mathrm{KIM}^{3}$, Jinsil SEONG ${ }^{4}$ \\ ${ }^{1}$ Department of Surgery, Yongin Severance Hospital, Yonsei University College of Medicine, Yongin, Korea \\ ${ }^{2}$ Division of Hepatobiliary and Pancreatic Surgery, Department of Surgery, Yonsei University College of Medicine, Seoul, Korea \\ ${ }^{3}$ Department of Internal Medicine, Yonsei University College of Medicine, Seoul, Korea \\ ${ }^{4}$ Departments of Radiation Oncology, Yonsei University College of Medicine, Seoul, Korea
}

Introduction: Liver resection is usually recommended in patients after downstaging for locally advanced hepatocellular carcinoma (HCC) to induce complete remission. However, the liver resection requirement in patients expected to have complete pathological necrosis (CPN) after HCC downstaging is questionable.

Methods: From 2002 to 2019, 919 patients with locally advanced HCC underwent concurrent chemoradiotherapy or transarterial radioembolization. Among these patients, excluding liver transplantation, 94 patients who underwent hepatic resection (OP group) and 789 patients who did not undergo surgical treatment (non-OP group) were included in this study. Predictive factors of CPN in the resected specimen after tumor downstaging in the OP group analyzed by logistic regression analysis.

Results: Of the 94 patients in the OP group, thirty-eight patients (40.4\%) were found to have CPN. In multivariable analysis, the predictive factors of CPN were complete radiologic response and tumor marker responder (hazard ratio [HR] 1.00, $p<0.006$; HR 3.698, $95 \%$ confidence interval $1.029-13.321, p=0.045)$. Among the non-OP group, $11 \%$ of patients belonged to the CPN-predictive factor (PF) group. Of these patients, only two patients (18.2\%) have occurred intrahepatic recurrence. There was no difference in disease-free survival between the CPN-PF group and the CPN group (119.0 \pm 77.42 vs. $60.00 \pm 14.04, p=0.075)$. In addition, the overall survival (OS) of the CPN-PF group was significantly higher than the OS of the CPN group (171.00 \pm 0.00 vs. $97.00 \pm 17.46, p=0.044)$.

Conclusions: This study showed that surgical resection might not provide further advantages for long-term outcomes in patients with CPN-PFs after HCC downstaging. 